\title{
Modelagem e Simulação dos Efeitos Térmicos de um Reator de Leito Fixo para Reforma do Alcatrão para Geração de Gás Combustível
}

Silva, F. R.

Escola Politécnica de Pernambuco Universidade de Pernambuco 50.720-001 - Recife, Brasil Autor1@meu-email
Silva, J. D.

Escola Politécnica de Pernambuco Universidade de Pernambuco 50.720-001 - Recife, Brasil Autor2@meu-email

\section{Autor 3}

Escola Politécnica de Pernambuco Universidade de Pernambuco 50.720-001 - Recife, Brasil Autor2@meu-email

Resumo O alcatrão formado durante o processo de gaseificação de biomassa é um composto indesejável que prejudica o rendimento do processo e danifica equipamentos. Os métodos de eliminação do alcatrão podem ser divididos em primários e secundários, sendo os primários os métodos cataliticos, e os secundários os métodos de limpeza do gás produzido. O foco deste trabalho é o estudo da eliminação do alcatrão através do processo catalítico utilizando-se um catalisador a base de níquel. Através do modelo matemático proposto, os componentes do processo de craqueamento do alcatrão serão analisados, quantificando-se produção e consumo e o comportamento das temperaturas nas fases gasosa e sólida para um reator de leito fixo.

\footnotetext{
Abstract The tar formed during the gasification of biomass is an undesirable compound that interferes with the performance of the process and damage equipment. The methods of disposal of tar can be divided into primary and secondary, with the primary catalytic methods, and secondary methods of cleaning the gas produced. The focus of this work is to study the removal of tar through the catalytic process using a catalyst based on nickel. Through the mathematical model, the components of the tar cracking process will be analyzed, quantifying the production and consumption and the behavior of temperatures in gaseous and solid phases for a fixed bed reactor..
} 


\section{Introdução}

A gaseificação de biomassa é um importante processo de aproveitamento de biomassa para a produção de combustíveis e produtos químicos. Durante a gaseificação, a biomassa, através de reações com ar, oxigênio ou vapor de água é convertida em uma mistura de gases como $\mathrm{CO}_{2}$ $\mathrm{CO}, \mathrm{H}_{2}, \mathrm{H}_{2} \mathrm{O}$. Além dos gases há também a formação de uma pequena quantidade de hidrocarbonetos voláteis juntamente com o alcatrão [1]. O alcatrão formado durante o processo de gaseificação pode ser definido como todo contaminante orgânico com peso molecular maior do que o do benzeno [2]. Porém, esta é apenas uma das diversas definições dadas a este composto, já que para defini-lo com base em sua composição química fatores como variação da composição do produto gasoso final desejado para uma aplicação particular e de como o alcatrão é coletado e analisado devem ser levados em consideração. O alcatrão é um composto indesejável, pois pode condensar ou polimerizar-se em estruturas mais complexas nas tubulações, filtros e trocadores de calor, danificando equipamentos, diminuindo a eficiência total e aumentando o custo do processo [3]. O principal método de remoção do alcatrão formado durante o processo de gaseificação de biomassa é a reforma catalítica, os tipos de catalisadores mais amplamente pesquisados e desenvolvidos são a dolomita calcinada, olivina e catalisadores a base de $\mathrm{Ni}$, sendo o mais promissor o catalisador a base de níquel. O objetivo deste trabalho é caracterizar o craqueamento do alcatrão através do catalisador a base de níquel. Para isso será considerada a seguinte reação:

$$
2 \mathrm{C}_{7} \mathrm{H}_{8}+7 \mathrm{O}_{2} \stackrel{\mathrm{Ni}^{2} / \mathrm{Al}_{2} \mathrm{O}_{3}}{\longrightarrow} 8 \mathrm{H}_{2}+14 \mathrm{CO}
$$

O tolueno foi usado como composto modelo de alcatrão, porque representa uma estrutura aromática estável resultante em alcatrões formados em processo a altas temperaturas [4]. Através do modelo matemático desenvolvido serão analisados os perfis de temperatura e de transferência de massa para todos os componentes da reação.

\section{Modelagem Matemática}

Neste trabalho o modelo matemático para a reforma catalítica do alcatrão foi desenvolvido através das equações de conservação de energia e massa, assim desenvolveu-se um conjunto de equações que caracteriza a transferência de energia e massa para o fenômeno da reforma do alcatrão cuja solução descreve os perfis de temperatura e fração molar para todos os componentes da reação estudada. As equações gerais que descrevem o fenômeno da reforma catalítica do alcatrão são as seguintes:

- Balanço de energia para a fase vapor:

$$
\begin{aligned}
& \left(1-\varepsilon_{\mathrm{s}}\right) \rho_{\mathrm{v}} \mathrm{C}_{\mathrm{p}, \mathrm{v}} \frac{\partial \mathrm{T}_{\mathrm{v}}}{\partial \mathrm{t}}+\rho_{\mathrm{v}} \mathrm{C}_{\mathrm{p}, \mathrm{v}} \mathrm{V}_{\mathrm{v}} \frac{\partial \mathrm{T}_{\mathrm{v}}}{\partial \mathrm{z}}= \\
& \left(1-\varepsilon_{\mathrm{s}}\right) \lambda_{\mathrm{ax}, \mathrm{v}} \frac{\partial^{2} \mathrm{~T}_{\mathrm{v}}}{\partial \mathrm{z}^{2}}-\left(\alpha_{\mathrm{vs}} \eta_{\mathrm{CE}} \varepsilon_{\mathrm{s}} \mathrm{a}_{\mathrm{s}}\right)\left[\mathrm{T}_{\mathrm{v}}-\mathrm{T}_{\mathrm{s}}\right] \\
& +\alpha_{\mathrm{vw}}\left(1-\eta_{E C}\right) \mathrm{a}_{\mathrm{w}}\left[\mathrm{T}_{\mathrm{v}}-\mathrm{T}_{\mathrm{w}}\right]
\end{aligned}
$$

- Condições iniciais e de contorno:

$$
\begin{aligned}
& \left.\mathrm{T}_{\mathrm{v}}\right|_{\mathrm{t}=0}=\mathrm{T}_{\mathrm{v}, \mathrm{e}} \\
& \left.\left(1-\varepsilon_{\mathrm{s}}\right) \lambda_{\mathrm{ax}, \mathrm{v}} \frac{\partial \mathrm{T}_{\mathrm{v}}}{\partial \mathrm{z}}\right|_{\mathrm{z}=0^{+}}= \\
& \rho_{\mathrm{v}} \mathrm{C}_{\mathrm{p}, \mathrm{v}} \mathrm{V}_{\mathrm{v}}\left[\left.\mathrm{T}_{\mathrm{v}}\right|_{\mathrm{z}=0^{+}}-\mathrm{T}_{\mathrm{v}, \mathrm{e}}\right] \\
& \left.\frac{\partial \mathrm{T}_{\mathrm{v}}}{\partial \mathrm{z}}\right|_{\mathrm{z}=\mathrm{L}}=0
\end{aligned}
$$

- Balanço de energia para a fase sólida:

$$
\begin{aligned}
& \varepsilon_{\mathrm{s}} \rho_{\mathrm{s}} \mathrm{C}_{\mathrm{p}, \mathrm{s}} \frac{\partial \mathrm{T}_{\mathrm{s}}}{\partial \mathrm{t}}=\varepsilon_{\mathrm{s}} \lambda_{\mathrm{ax}, \mathrm{s}} \frac{\partial^{2} \mathrm{~T}_{\mathrm{s}}}{\partial \mathrm{z}^{2}} \\
& +\left(\alpha_{\mathrm{vs}} \mathrm{M}_{\mathrm{CE}} \varepsilon_{\mathrm{s}} \mathrm{a}_{\mathrm{s}}\right)\left[\mathrm{T}_{\mathrm{v}}-\mathrm{T}_{\mathrm{s}}\right]+\varepsilon_{\mathrm{s}} \mathrm{r}_{\mathrm{x}}^{\prime}\left(-\Delta \mathrm{H}_{\mathrm{r}}\right)
\end{aligned}
$$

- Condições iniciais e de contorno:

$$
\begin{aligned}
& \left.\mathrm{T}_{\mathrm{s}}\right|_{\mathrm{t}=0}=\mathrm{T}_{\mathrm{s}, \mathrm{e}} \\
& \left.\varepsilon_{\mathrm{s}} \lambda_{\mathrm{ax}, \mathrm{s}} \frac{\partial \mathrm{T}_{\mathrm{s}}}{\partial \mathrm{z}}\right|_{\mathrm{z}=0^{+}}=\mathrm{q}_{\mathrm{c}}
\end{aligned}
$$


$\left.\frac{\partial \mathrm{T}_{\mathrm{s}}}{\partial \mathrm{z}}\right|_{\mathrm{z}=\mathrm{L}}=0$

- Balanço de massa para o tolueno:

$\mathrm{h}_{\mathrm{t}} \frac{\partial \mathrm{C}_{\mathrm{t}}}{\partial \mathrm{t}}+\frac{4 \mathrm{Q}_{\mathrm{g}}}{\pi \mathrm{d}^{2}} \frac{\partial \mathrm{C}_{\mathrm{t}}}{\partial \mathrm{z}}=\mathrm{D}_{\mathrm{t}} \frac{\partial^{2} \mathrm{C}_{\mathrm{t}}}{\partial \mathrm{z}^{2}}$

$+\varepsilon_{\mathrm{s}} \mathrm{M}_{\mathrm{t}} \mathrm{R}_{\mathrm{t}}$

- Condições iniciais e de contorno para o tolueno:

$$
\begin{aligned}
& \left.\mathrm{C}_{\mathrm{t}}\right|_{\mathrm{t}=0}=\mathrm{C}_{\mathrm{t}, 0} \\
& \left.\mathrm{D}_{\mathrm{t}} \frac{\partial \mathrm{C}_{\mathrm{t}}}{\partial \mathrm{z}}\right|_{\mathrm{z}=0^{+}}=\frac{4 \mathrm{Q}_{\mathrm{g}}}{\pi \mathrm{d}^{2}}\left[\left.\mathrm{C}_{\mathrm{t}}\right|_{z=0^{+}}-\mathrm{C}_{\mathrm{t}, 0}\right] \\
& \left.\frac{\partial \mathrm{C}_{\mathrm{t}}}{\partial \mathrm{z}}\right|_{z=1}=0
\end{aligned}
$$

- Balanço de massa para o $\mathrm{O}_{2}$ :

$$
\begin{aligned}
& \mathrm{h}_{\mathrm{t}} \frac{\partial \mathrm{C}_{\mathrm{O}_{2}}}{\partial \mathrm{t}}+\frac{4 \mathrm{Q}_{\mathrm{g}}}{\pi \mathrm{d}^{2}} \frac{\partial \mathrm{C}_{\mathrm{O}_{2}}}{\partial \mathrm{z}}=\mathrm{D}_{\mathrm{O}_{2}} \frac{\partial^{2} \mathrm{C}_{\mathrm{O}_{2}}}{\partial \mathrm{z}^{2}} \\
& +\varepsilon_{\mathrm{s}} \mathrm{M}_{\mathrm{O}_{2}} \mathrm{R}_{\mathrm{O}_{2}}
\end{aligned}
$$

- Condições iniciais e de contorno para o $\mathrm{O}_{2}$ :

$$
\begin{aligned}
& \left.\mathrm{C}_{\mathrm{O}_{2}}\right|_{\mathrm{t}=0}=\mathrm{C}_{\mathrm{O}_{2}, 0} \\
& \left.\mathrm{D}_{\mathrm{O}_{2}} \frac{\partial \mathrm{C}_{\mathrm{O}_{2}}}{\partial \mathrm{z}}\right|_{\mathrm{z}=0^{+}}=\frac{4 \mathrm{Q}_{\mathrm{g}}}{\pi \mathrm{d}^{2}}\left[\left.\mathrm{C}_{\mathrm{O}_{2}}\right|_{\mathrm{z}=0^{+}}-\mathrm{C}_{\mathrm{O}_{2}, 0}\right] \\
& \left.\frac{\partial \mathrm{C}_{\mathrm{O}_{2}}}{\partial \mathrm{z}}\right|_{\mathrm{z}=1}=0
\end{aligned}
$$

- Balanço de massa para o CO:

$$
\begin{aligned}
& \mathrm{h}_{\mathrm{t}} \frac{\partial \mathrm{C}_{\mathrm{CO}}}{\partial \mathrm{t}}+\frac{4 \mathrm{Q}_{\mathrm{g}}}{\pi \mathrm{d}^{2}} \frac{\partial \mathrm{C}_{\mathrm{CO}}}{\partial \mathrm{z}}=\mathrm{D}_{\mathrm{CO}} \frac{\partial^{2} \mathrm{C}_{\mathrm{CO}}}{\partial \mathrm{z}^{2}} \\
& +\varepsilon_{\mathrm{s}} \mathrm{M}_{\mathrm{CO}} \mathrm{R}_{\mathrm{CO}}
\end{aligned}
$$

- Condições iniciais e de contorno para o CO:

$$
\begin{aligned}
& \left.\mathrm{C}_{\mathrm{CO}}\right|_{\mathrm{t}=0}=\mathrm{C}_{\mathrm{CO}, 0} \\
& \left.\mathrm{D}_{\mathrm{CO}} \frac{\partial \mathrm{C}_{\mathrm{CO}}}{\partial \mathrm{z}}\right|_{z=0^{+}}=\frac{4 \mathrm{Q}_{\mathrm{g}}}{\pi \mathrm{d}^{2}}\left[\left.\mathrm{C}_{\mathrm{CO}}\right|_{z=0^{+}}-\mathrm{C}_{\mathrm{CO}, 0}\right] \\
& \left.\frac{\partial \mathrm{C}_{\mathrm{CO}}}{\partial \mathrm{z}}\right|_{z=1}=0
\end{aligned}
$$

- Balanço de massa para o $\mathrm{H}_{2}$ :

$$
\begin{aligned}
& \mathrm{h}_{\mathrm{t}} \frac{\partial \mathrm{C}_{\mathrm{H}_{2}}}{\partial \mathrm{t}}+\frac{4 \mathrm{Q}_{\mathrm{g}}}{\pi \mathrm{d}^{2}} \frac{\partial \mathrm{C}_{\mathrm{H}_{2}}}{\partial \mathrm{z}}=\mathrm{D}_{\mathrm{H}_{2}} \frac{\partial^{2} \mathrm{C}_{\mathrm{H}_{2}}}{\partial \mathrm{z}^{2}}+ \\
& \varepsilon_{\mathrm{s}} \mathrm{M}_{\mathrm{H}_{2}} \mathrm{R}_{\mathrm{H}_{2}}
\end{aligned}
$$

- Condições iniciais e de contorno para $\mathrm{O}_{2}$ : 


$$
\begin{aligned}
& \left.\mathrm{C}_{\mathrm{H}_{2}}\right|_{\mathrm{t}=0}=\mathrm{C}_{\mathrm{H}_{2}, 0} \\
& \left.\mathrm{D}_{\mathrm{H}_{2}} \frac{\partial \mathrm{C}_{\mathrm{H}_{2}}}{\partial \mathrm{z}}\right|_{\mathrm{z}=0^{+}}=\frac{4 \mathrm{Q}_{\mathrm{g}}}{\pi \mathrm{d}^{2}}\left[\left.\mathrm{C}_{\mathrm{H}_{2}}\right|_{\mathrm{z}=0^{+}}-\mathrm{C}_{\mathrm{H}_{2}, 0}\right] \\
& \left.\frac{\partial \mathrm{C}_{\mathrm{H}_{2}}}{\partial \mathrm{z}}\right|_{\mathrm{z}=1}=0
\end{aligned}
$$

As equações de (1) a (26) podem ser simplificadas segundo as seguintes hipóteses simplificadoras: (i) modelo unidimensional, (ii) sistema não-isotérmico, (iii) as temperaturas da fase gasosa e sólida são modeladas através de modelos de dispersão térmica e (iv) os balanços de massa para a fase gasosa e sólida são modelados levando-se em consideração os efeitos de dispersão axial. Baseado nessas hipóteses, o modelo matemático simplificado para a reforma catalítica do alcatrão é formado pelas equações simplificadas de transporte energia e massa que descrevem o comportamento das temperaturas e frações molares. Assim, as equações simplificadas que formam o modelo são expressas por:

- Balanço de energia para a fase vapor:

$$
\begin{aligned}
& \frac{\mathrm{dT}_{\mathrm{v}}}{\mathrm{dt}}=\alpha_{13, \mathrm{v}}\left(\mathrm{T}_{\mathrm{v}}\right)_{\mathrm{j}}^{(\mathrm{k})}+\alpha_{3, \mathrm{v}}\left(\mathrm{T}_{\mathrm{s}}\right)_{\mathrm{j}}^{(\mathrm{k})}- \\
& \alpha_{4, \mathrm{v}}\left(\mathrm{T}_{\mathrm{w}}\right)_{\mathrm{j}}^{(\mathrm{k})}+\alpha_{14, \mathrm{v}}
\end{aligned}
$$

- Balanço de energia para a fase sólida:

$$
\frac{\mathrm{dT}_{\mathrm{s}}}{\mathrm{dt}}=\alpha_{21, \mathrm{~s}}\left(\mathrm{~T}_{\mathrm{s}}\right)_{\mathrm{j}}^{(\mathrm{k})}+\alpha_{16, \mathrm{~s}}\left(\mathrm{~T}_{\mathrm{v}}\right)_{\mathrm{j}}^{(\mathrm{k})}+\alpha_{22, \mathrm{~s}}
$$

- Balanço de massa para o tolueno:

$$
\frac{\mathrm{dC}_{\mathrm{t}}}{\mathrm{dt}}=\alpha_{12, \mathrm{t}}\left(\mathrm{C}_{\mathrm{t}}\right)_{\mathrm{j}}^{(\mathrm{k})}+\alpha_{13, \mathrm{t}}+\alpha_{2, \mathrm{t}}
$$

- Balanço de massa para o $\mathrm{O}_{2}$ :

$$
\frac{\mathrm{dC}_{\mathrm{O}_{2}}}{\mathrm{dt}}=\alpha_{12, \mathrm{O}_{2}}\left(\mathrm{C}_{\mathrm{O}_{2}}\right)_{\mathrm{j}}^{(\mathrm{k})}+\alpha_{13, \mathrm{O}_{2}}+\alpha_{2, \mathrm{O}_{2}}
$$

- Balanço de massa para o CO

$$
\frac{\mathrm{dC}_{\mathrm{CO}}}{\mathrm{dt}}=\alpha_{12, \mathrm{CO}}\left(\mathrm{C}_{\mathrm{CO}}\right)_{\mathrm{j}}^{(\mathrm{k})}+\alpha_{13, \mathrm{CO}}+\alpha_{2, \mathrm{CO}}
$$

- Balanço de massa para o $\mathrm{H}_{2}$ :

$$
\frac{\mathrm{dC}_{\mathrm{H}_{2}}}{\mathrm{dt}}=\alpha_{12, \mathrm{H}_{2}}\left(\mathrm{C}_{\mathrm{H}_{2}}\right)_{\mathrm{j}}^{(\mathrm{k})}+\alpha_{13, \mathrm{H}_{2}}+\alpha_{2, \mathrm{H}_{2}}
$$

A simplificação das equações gerou um conjunto de parâmetros $\alpha$ que estão apresentados nas tabelas 1 a 5 do apêndice $\mathrm{A}$.

\section{Resultados e Discussões}

Nesta seção, os resultados da simulação do modelo serão apresentados. A resolução do modelo mostra o perfil

\begin{tabular}{|c|c|}
\hline$\varepsilon_{g}=0,72$ & $a_{w}=0,57 \mathrm{~m}^{2}$ \\
\hline$\rho_{\mathrm{v}}=0,956 \mathrm{~kg} / \mathrm{m}^{3}$ & $\mathrm{q}_{\mathrm{c}}=208 \mathrm{~kJ} / \mathrm{s}$ \\
\hline $\mathrm{C}_{\text {Rax }}=32 \mathrm{~J} / \mathrm{mol} \mathrm{K}$ & $h_{t}=0,46$ \\
\hline $\mathrm{V}_{\mathrm{V}}=0,021 \mathrm{~m} / \mathrm{s}$ & $\mathrm{Q}_{\mathrm{g}}=2,32 \times 10^{-3} \mathrm{~m}^{3} / \mathrm{s}$ \\
\hline$\lambda_{\mathrm{ax}, \mathrm{v}}=2,09 \mathrm{~J} / \mathrm{m}^{2} \mathrm{~s} \mathrm{~K}$ & $\mathrm{~d}=1 \mathrm{~m}$ \\
\hline$\alpha_{\mathrm{vs}}=1,81 \mathrm{~W} / \mathrm{m}^{2} \mathrm{~K}$ & $\mathrm{D}_{\mathrm{t}}=1,25 \times 10^{-5} \mathrm{~m}^{2} / \mathrm{s}$ \\
\hline$\eta_{\mathrm{ce}}=0,35$ & $\mathrm{D}_{\mathrm{O} 2}=3,24 \times 10^{-5} \mathrm{~m}^{2} / \mathrm{s}$ \\
\hline$a_{\mathrm{s}}=156 \mathrm{~m}^{-1}$ & $\mathrm{D}_{c Q}=4,61 \times 10^{-6} \mathrm{~m}^{2} / \mathrm{s}$ \\
\hline$\rho_{\mathrm{s}}=1250 \mathrm{~kg} / \mathrm{m}^{3}$ & $\mathrm{D}_{\mathrm{H} 2}=6,52 \times 10^{-6} \mathrm{~m}^{2} / \mathrm{s}$ \\
\hline $\mathrm{C}_{\text {pdos }}=18 \mathrm{~J} / \mathrm{mol} \mathrm{K}$ & $\eta_{t}=0,95$ \\
\hline$\lambda_{\mathrm{ax}, \mathrm{s}}=1,79 \mathrm{~J} / \mathrm{m}^{2} \mathrm{~s} \mathrm{~K}$ & $\eta_{\mathrm{O} 2}=0,65$ \\
\hline$\Delta \mathrm{H}_{\mathrm{r}}=-283 \mathrm{~kJ} / \mathrm{mol}$ & $\eta_{\mathrm{CO}}=0,79$ \\
\hline$\alpha_{\mathrm{vw}}=0,12$ & $\eta_{\mathrm{H} 2}=0,86$ \\
\hline
\end{tabular}
do comportamento da temperatura nas fases gasosa e sólida e fração molar para todos os componentes envolvidos na reação de reforma catalítica do alcatrão. Estas equações foram resolvidas usando o método Runge Kutta Gill, na sequência, foi desenvolvido um programa computacional na linguagem FORTRAN para fornecer os resultados das variáveis $\mathrm{T}_{\mathrm{g}}, \mathrm{T}_{\mathrm{s}}, \mathrm{C}_{7} \mathrm{H}_{8}, \mathrm{O}_{2}, \mathrm{CO}$ e $\mathrm{H}_{2}$. O programa recebeu parâmetros de experimentos independentes mostrados na Tabela 1. 
Os resultados serão mostrados em sua forma gráfica. A figura 1 caracteriza os perfis de temperatura para as fases gasosa e sólida, sendo $\mathrm{O}$ aumento da temperatura tanto da fase sólida como da fase gasosa devido à agitação molecular dentro do gaseificador.

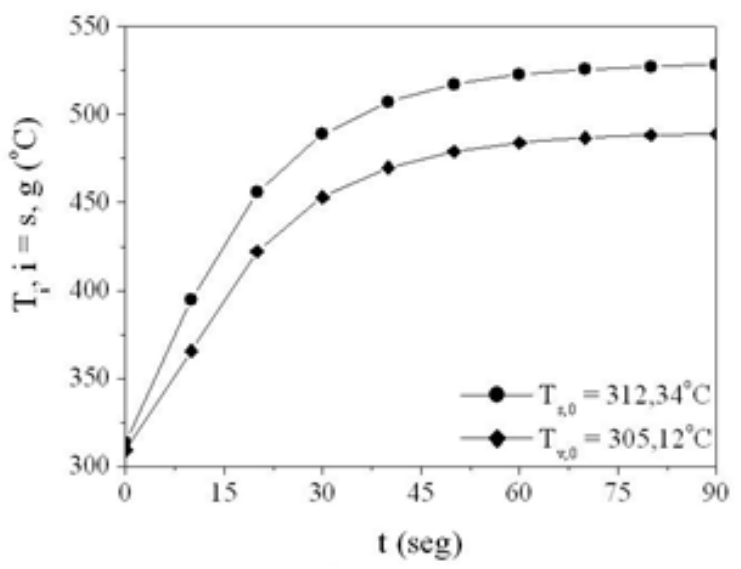

Fig 1. Perfis de temperatura para a fase vapor $\left(T_{v}\right)$ e para a fase sólida $\left(T_{s}\right)$

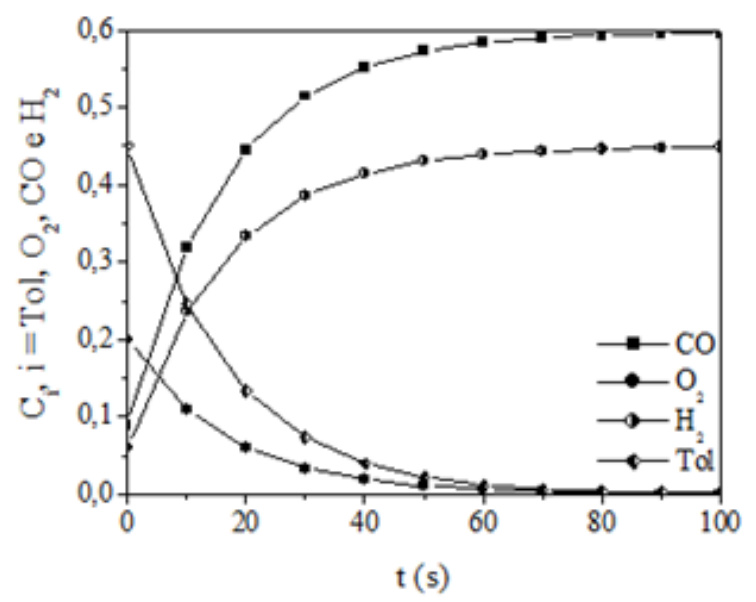

Fig 2. Perfis de concentrações das espécies químicas $\mathrm{C}_{7} \mathrm{H}_{8}, \mathrm{O}_{2}, \mathrm{CO}$ e $\mathrm{H}_{2}$, na saída do reator catalítico trifásico de leito fixo.

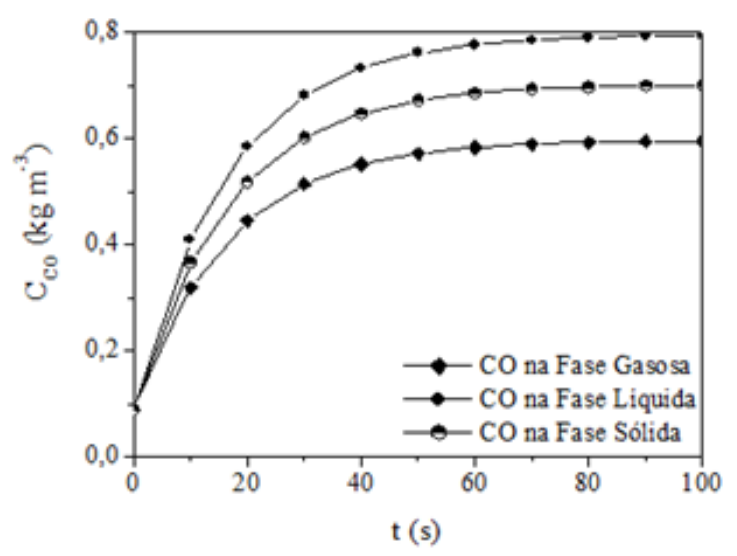

Fig 3. Perfis da concentração do componente $\mathrm{CO}$ nas fases gasosa, líquida e sólida do reator trifásico catalítico de leito fixo

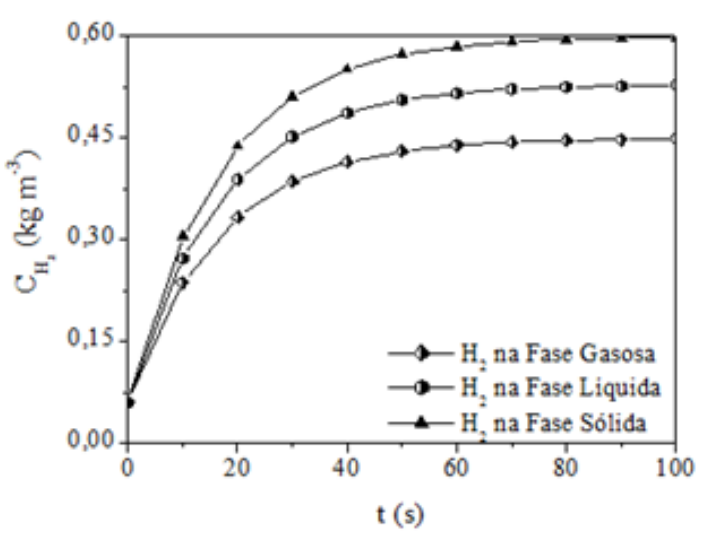

Fig 4. Perfis da concentração do componente $\mathrm{H}_{2}$ nas fases gasosa, líquida e sólida

\section{Conclusões}

O estudo mostrado no presente trabalho permitiu analisar os comportamentos da temperatura do fluido gasoso, da temperatura na fase sólida e da fração molar das espécies químicas $\mathrm{C}_{7} \mathrm{H}_{8}, \mathrm{O}_{2}$, $\mathrm{CO}$ e $\mathrm{H}_{2}$. A partir dos resultados mostrados, pode-se apresentar as seguintes conclusões:

- A temperatura da fase vapor aumenta estabilizando-se em $\pm 487,5^{\circ} \mathrm{C}$;

- A temperatura da fase sólida aumenta estabilizase em $\pm 525^{\circ} \mathrm{C}$;

- A concetração de CO cresce conforme o tolueno e $\mathrm{o}_{2}$ são consumidos e estabiliza-se em $\pm 0,6$ $\mathrm{Kg} m^{-3}$; 
- A concentração de $\mathrm{H}_{2}$ cresce conforme o tolueno e o $\mathrm{O}_{2}$ são consumidos e estabiliza-se em $\pm 0,45$ $\mathrm{Kg} m^{-3}$;

\section{Notoções}

$\mathrm{C}_{\mathrm{p}, \mathrm{v}}$ Capacidade calorífica da fase vapor de tolueno, $\mathrm{J} / \mathrm{mol} \mathrm{K}$

$\mathrm{C}_{\mathrm{p}, \mathrm{s}} \quad$ Capacidade calorífica da fase sólida, $\mathrm{J} / \mathrm{mol} \mathrm{K}$

$\mathrm{V}_{\mathrm{v}}$ Velocidade da fase vapor, $\mathrm{m} / \mathrm{s}$

$\mathrm{a}_{\mathrm{S}}$ Área específica por volume, $\mathrm{m}^{2} / \mathrm{m}^{3}$

$\mathrm{T}_{\mathrm{v}}$ Temperatura da fase vapor, $\mathrm{K}$

$\mathrm{T}_{\mathrm{s}}$ Temperatura da fase sólida, $\mathrm{K}$

$\mathrm{T}_{\mathrm{w}}$ Temperatura da parede, $\mathrm{K}$

$\mathrm{T}_{v, l} \quad$ Temperatura do vapor na saída do reator, $\mathrm{K}$

$\mathrm{a}_{\mathrm{w}}$ Área da parede do reator, $\mathrm{m}^{2}$

$\mathrm{r}_{\mathrm{x}}$ Taxa de reação, $\mathrm{mol} / \mathrm{s} \mathrm{m}^{3}$

$-\Delta \mathrm{H}_{\mathrm{r}}$ Entalpia da reação,k $\mathrm{J} / \mathrm{mol}$

$\mathrm{q}_{\mathrm{c}}$ Fluxo de calor na entrada do reator, $\mathrm{kJ} / \mathrm{s}$

$h_{t}$ Coeficiente de retenção do tolueno, (-)

$\mathrm{h}_{\mathrm{O}_{2}} \quad$ Coeficiente de retenção do oxigênio, (-)

$\mathrm{h}_{\mathrm{CO}}$ Coeficiente de retenção do óxido de carbono, (-)

$h_{\mathrm{H}_{2}}$ Coeficiente de retenção do hidrogênio, (-)

$\mathrm{Q}_{\mathrm{g}}$ Vazão do gás, $\mathrm{m}^{3} / \mathrm{s}$

d Diâmetro do reator, $m$

$\mathrm{D}_{\mathrm{t}}$ Coeficiente molecular de difusão do tolueno, $\mathrm{m}^{2} / \mathrm{s}$

$\mathrm{D}_{\mathrm{O}_{2}}$ Coeficiente molecular de difusão do oxigênio, $\mathrm{m}^{2} / \mathrm{s}$

$\mathrm{D}_{\mathrm{CO}}$ Coeficiente molecular de difusão do óxido de carbono, $\mathrm{m}^{2} / \mathrm{s}$

$\mathrm{D}_{\mathrm{H}_{2}}$ Coeficiente molecular de difusão do hidrogênio, $\mathrm{m}^{2} / \mathrm{s}$

$\mathrm{R}_{\mathrm{t}}$ Taxa de reação para o tolueno, $1 / \mathrm{s}$

$\mathrm{R}_{\mathrm{O}_{2}}$ Taxa de reação para o oxigênio, $1 / \mathrm{s}$

$\mathrm{R}_{\mathrm{CO}}$ Taxa de reação para o monóxido de carbono, 1/s

$\mathrm{R}_{\mathrm{H}_{2}}$ Taxa de reação para o hidrogênio, 1/s

\section{Letras Gregas}

$\varepsilon_{\mathrm{S}}$ Porosidade da fase sólida, (-)

$\rho_{\mathrm{v}}$ Densidade da fase vapor de tolueno, $\mathrm{Kg} / \mathrm{m}^{3}$

$\rho_{\mathrm{s}}$ Densidade da fase sólida, $\mathrm{Kg} / \mathrm{m}^{3}$

$\lambda_{\text {ax,v }}$ Dispersão térmica do vapor de tolueno, $\mathrm{J} / \mathrm{m}^{2} \mathrm{~s} \mathrm{~K}$

$\lambda_{\text {ax,s }}$ Dispersão térmica da fase sólida, $\mathrm{J} / \mathrm{m}^{2} \mathrm{~s} \mathrm{~K}$ $\alpha_{v, s} \quad$ Coeficiente de transferência vapor de tolueno-só-

lido, $\mathrm{W} / \mathrm{m}^{2} \mathrm{~K}$

$\eta_{\mathrm{CE}} \quad$ Eficiência de molhabilidade externa, (-)

$\eta_{\mathrm{t}}$ Fator de efetividade do tolueno, (-)

$\eta_{\mathrm{O}_{2}}$ Fator de efetividade do oxigênio, (-)

$\eta_{\mathrm{CO}}$ Fator de efetividade do monóxido de carbono, (-)

$\eta_{\mathrm{H}_{2}} \quad$ Fator de efetividade do hidrogênio, (-)

$\alpha_{v, w}$ Coeficiente de transferência na parede, (-)

\section{Agradecimentos}

Os autores deste artigo gostariam de agradecer ao CNPQ (Conselho Nacional de Desenvolvimento Científico e Tecnológico) pelo suporte financeiro dado. (Processo 48354 / 2007 / Projeto / Título: Modelagem Matemática, Simulação Computacional e Otimização dos Fenômenos de Transferência de Energia e Massa para um Reator Trifásico de Leito Fixo para a Reforma do Alcatrão./ Edital CNPq 15 / 2007 - Universal).

\section{Referências}

[1] Sundac, Nikola., Catalytic Cracking of tar from biomass gasification. Department of Chemical Engineering,, Lund University, P. O. Box 124, SE-22100 Lund, Sweden

[2] Devi, L., Ptasinski, K.J., Janssen, F.J.J.G, A review of primary measures for tar elimination in biomass gasification process, Biomass and Bioenergy, Vol. 24., pp. 125-140 (2003).

[3] Lamacz, A.; Krzton, A.; Musi, A.; Da Costa, P.; Reforming of Model Gasification Tar Compounds. Catalysis Letters, V. 128, Numbers 1-2, pp. 40-48.

[4] Swierczynski, D.; Courson, C.; Kiennemann, A Study of steam reforming of toluene used as model compound of tar produced by biomass gasification. Chemical Engineering and Processing, v. 47 , p. 508-513, 2007.

[5] Milne T.A., Evans R.J., Biomass Gasifier "Tars": Their Nature, Formation and Conversion, NREL/TP-570-25357(1998).

[6] Castro, A.J.A., Cassiano, D.A., Ferreira, N.L., Gaseificação do bagaço de cana-de-açucar: modelagem, análise e comparação com sistema real. Exacta, v. 7, pp 39-47 (2009). 


\section{Apêndice A}

Tabela A1: Parâmetros $\alpha$ para o sistema de equações

\begin{tabular}{|c|c|}
\hline$\alpha_{1, v}=\frac{V_{v}}{\left(1-\varepsilon_{s}\right)}$ & $\alpha_{12, v}=\frac{2 \Delta z \alpha_{8, v} T_{v, \theta}}{2-\Delta z \alpha_{8, v}}$ \\
\hline$\alpha_{2, v}=\frac{\lambda_{2 x, v}}{\rho_{v} C_{p, v}}$ & $\begin{array}{l}\alpha_{13,2}=\alpha_{9,2} \alpha_{9,2}+\alpha_{6,2} \alpha_{11,2} \\
+\alpha_{7,2}\end{array}$ \\
\hline$\alpha_{3, v}=\frac{\alpha_{v s} \eta_{C E} \varepsilon_{s} a_{3}}{\left(1-\varepsilon_{s}\right) \rho_{v} C_{p, v}}$ & $\begin{array}{l}\alpha_{14, v}=\alpha_{6, v} \alpha_{12, v}- \\
\alpha_{5, v} \alpha_{10, v}\end{array}$ \\
\hline$\alpha_{4, v}=\frac{\alpha_{v, w}\left(1-\eta_{E C}\right) a_{m}}{\left(1-\varepsilon_{s}\right) \rho_{v} C_{\rho, v}}$ & $\alpha_{15, s}=\frac{\lambda_{2 x, s}}{\rho_{3} C_{p, s}}$ \\
\hline$\alpha_{5, v}=\frac{\alpha_{2, v}}{(\Delta z)^{2}}-\frac{\alpha_{1, v}}{2 \Delta z}$ & $\alpha_{16, s}=\frac{\alpha_{v, s} \eta_{C E} a_{s}}{\rho_{s} C_{p s}}$ \\
\hline$\alpha_{6, v}=\frac{\alpha_{2, v}}{(\Delta z)^{2}}+\frac{\alpha_{1, v}}{2 \Delta z}$ & $\alpha_{17, s}=\frac{r_{x}^{\prime}\left(-\Delta H_{s}\right)}{\rho_{s} C_{p, s}}$ \\
\hline
\end{tabular}

\begin{tabular}{|c|c|}
\hline $\begin{array}{l}\alpha_{7, v}=\alpha_{4, v}-\alpha_{3, v}- \\
\frac{2 \alpha_{2 v}}{(\Delta z)^{2}}\end{array}$ & $\alpha_{18, s}=\frac{\alpha_{15, s}}{(\Delta Z)^{2}}$ \\
\hline$\alpha_{s, v}=\frac{\rho_{v} C_{p, v} V_{v}}{\left(1-\varepsilon_{s}\right) \lambda_{2 x, v}}$ & $\alpha_{19,3}=\left[\frac{-2 \alpha_{15,3}}{(\Delta Z)^{2}}-\alpha_{16,3}\right]$ \\
\hline$\alpha_{9, v}=\frac{2+\Delta z \alpha_{\Omega, v}}{2-\Delta z \alpha_{\Omega, v}}$ & $\alpha_{20, s}=\frac{-q_{c} \Delta Z}{\varepsilon_{s} \lambda_{2 x, s}}$ \\
\hline$\alpha_{10, v}=\frac{2 \Delta z \alpha_{\mathrm{g}, \mathrm{v}} \mathrm{T}_{\mathrm{v}, \mathrm{e}}}{2-\Delta z \alpha_{\mathrm{g}, \mathrm{v}}}$ & $\alpha_{21, s}=2 \alpha_{18,3}+\alpha_{19, s}$ \\
\hline$\alpha_{11, v}=\frac{2-\Delta z \alpha_{8, v}}{2+\Delta z \alpha_{8, v}}$ & $\begin{array}{l}\alpha_{22, z}=\alpha_{17, z}-\alpha_{18, z} \alpha_{20, \pm} \\
+\alpha_{18, z} \alpha_{21, z}\end{array}$ \\
\hline
\end{tabular}

Tabela A2: Parâmetros $\alpha_{\mathrm{t}}$ para o sistema de equações

\begin{tabular}{|c|c|}
\hline$a_{1, \mathrm{t}}=\frac{\mathrm{D}_{\mathrm{t}}}{\mathbf{h}_{\mathrm{t}}}$ & $\alpha_{j, t}=\frac{4 Q_{g}}{\pi d^{2} D_{t}}$ \\
\hline$\alpha_{2, t}=\frac{\varepsilon_{s} M_{t} R_{t}}{h_{t}}$ & $\alpha_{8, \mathrm{t}}=\left[\frac{2+\alpha_{7, t} \Delta z}{2-\alpha_{7, t} \Delta z}\right]$ \\
\hline$\alpha_{3, t}=\frac{4 Q_{g}}{h_{t} \pi d^{2}}$ & $\alpha_{9, t}=\left[\frac{2 \alpha_{9, t} \Delta z}{2-\alpha_{7, t} \Delta z}\right]$ \\
\hline$\alpha_{4, t}=\left[\frac{\alpha_{1, t}}{\Delta z^{2}}-\frac{\alpha_{3, t}}{2 \Delta z}\right]$ & $\alpha_{10, \mathrm{t}}=\left[\frac{2-\alpha_{7, \mathrm{t}} \Delta \mathrm{z}}{2+\alpha_{7, \mathrm{t}} \Delta z}\right]$ \\
\hline$\alpha_{S, t}=\left[\frac{\alpha_{1, t}}{\Delta z^{2}}+\frac{\alpha_{3, t}}{2 \Delta z}\right]$ & $\alpha_{11, \mathrm{t}}=\left[\frac{2 \alpha_{7, \mathrm{t}} \Delta \mathrm{z}}{2+\alpha_{\gamma, \mathrm{t}} \Delta z}\right.$ \\
\hline$\alpha_{6, t}=\frac{2 \alpha_{1, t}}{\Delta z^{2}}$ & $\begin{array}{l}\alpha_{12, t}=\alpha_{4, t} \alpha_{8, t}+ \\
\alpha_{5, t} \alpha_{10, t}-\alpha_{6, t}\end{array}$ \\
\hline$\alpha_{13, t}=\alpha$ & $-\alpha_{2, t}-\alpha_{4, t} \alpha_{9, t}$ \\
\hline
\end{tabular}

Tabela A3: Parâmetros $\alpha_{\mathrm{O}_{2}}$ para o sistema de equações

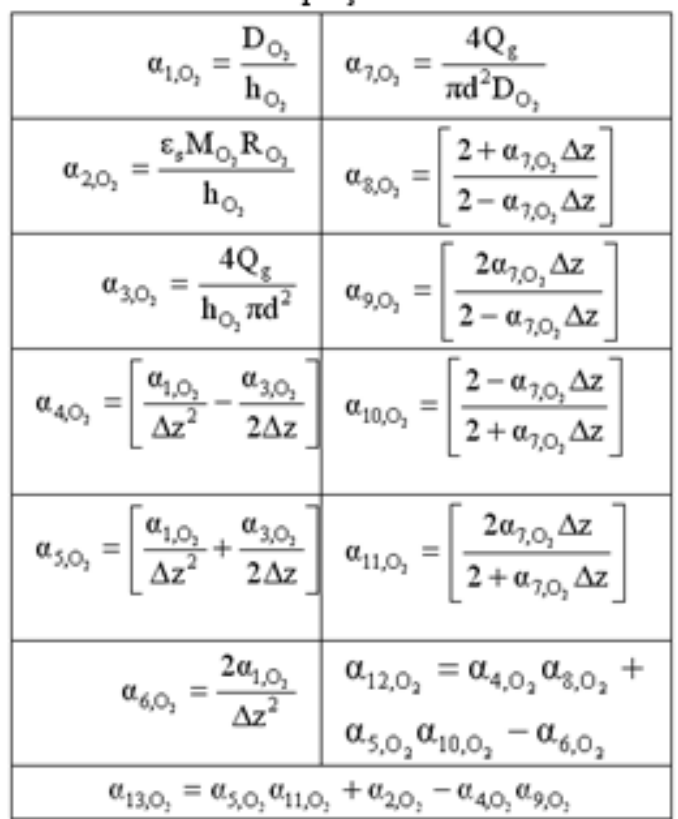


Tabela A4: Parâmetros $\alpha_{\mathrm{H}_{2}}$ para o sistema de equações

\begin{tabular}{|c|c|}
\hline$a_{1, H_{2}}=\frac{D_{H_{2}}}{h_{H_{2}}}$ & $\alpha_{7, \mathrm{H}_{2}}=\frac{4 \mathrm{Q}_{\mathrm{g}}}{\pi \mathrm{d}^{2} \mathrm{D}_{\mathrm{H}_{2}}}$ \\
\hline$\alpha_{2, \mathrm{H}_{2}}=\frac{\varepsilon_{t} \mathrm{M}_{\mathrm{H}_{2}} \mathrm{R}_{\mathrm{H}_{2}}}{\mathrm{~h}_{\mathrm{H}_{2}}}$ & $a_{8, \mathrm{H}_{2}}=\left[\frac{2+\alpha_{7, \mathrm{H}_{2}} \Delta z}{2-\alpha_{7, \mathrm{H}_{2}} \Delta z}\right]$ \\
\hline$\alpha_{3, H_{2}}=\frac{4 Q_{g}}{h_{H_{2}} \pi d^{2}}$ & $\alpha_{9, \mathrm{H}_{2}}=\left[\frac{2 \alpha_{7, \mathrm{H}_{2}} \Delta \mathrm{z}}{2-\alpha_{7, \mathrm{H}_{2}} \Delta \mathrm{z}}\right.$ \\
\hline$\alpha_{4, \mathrm{H}_{2}}=\left[\frac{\alpha_{1, \mathrm{H}_{2}}}{\Delta \mathrm{z}^{2}}-\frac{\alpha_{3, \mathrm{H}_{2}}}{2 \Delta \mathrm{z}}\right.$ & $\alpha_{10, H_{2}}=\left[\frac{2-\alpha_{7, H_{1}} \Delta z}{2+\alpha_{7, H_{3}} \Delta z}\right]$ \\
\hline$\alpha_{S, \mathrm{H}_{2}}=\left[\frac{\alpha_{1, \mathrm{H}_{2}}}{\Delta \mathrm{z}^{2}}+\frac{\alpha_{3, \mathrm{H}_{2}}}{2 \Delta \mathrm{z}}\right]$ & $\alpha_{11, \mathrm{H}_{2}}=\left[\frac{2 \alpha_{7, \mathrm{H}_{2}} \Delta z}{2+\alpha_{7, \mathrm{H}_{2}} \Delta z}\right]$ \\
\hline$\alpha_{6, \mathrm{H}_{2}}=\frac{2 \alpha_{1, \mathrm{H}_{2}}}{\Delta \mathrm{z}^{2}}$ & $\begin{array}{l}\alpha_{12, H_{2}}=\alpha_{4, H_{2}} \alpha_{8, H_{2}}+ \\
\alpha_{s, H_{2}} \alpha_{10, H_{2}}-\alpha_{6, H_{2}}\end{array}$ \\
\hline$\alpha_{13, \mathrm{H}_{2}}=\alpha_{\mathrm{S,}_{2}} \alpha_{11, h}$ & $+\alpha_{2, H_{2}}-\alpha_{4 H_{2}} \alpha_{9, H_{2}}$ \\
\hline
\end{tabular}

Tabela A5: Parâmetros $\alpha_{\text {co }}$ para o sistema de

\begin{tabular}{|c|c|}
\hline $\boldsymbol{\alpha}_{1, \infty 0}=\frac{\mathrm{D}_{\infty}}{\mathbf{h}_{\infty}}$ & $\alpha_{7, C O}=\frac{4 Q_{g}}{\pi d^{2} D_{C O}}$ \\
\hline$\alpha_{2, \infty O}=\frac{\varepsilon_{s} \mathrm{M}_{\infty} \mathrm{R}_{\infty}}{\mathbf{h}_{\infty}}$ & $\alpha_{8, \infty 0}=\left[\frac{2+\alpha_{7, \infty \infty} \Delta z}{2-\alpha_{3, \infty 0} \Delta z}\right]$ \\
\hline$\alpha_{3, C O}=\frac{4 Q_{g}}{h_{C O} \pi d^{2}}$ & $\alpha_{9, \infty 0}=\left[\frac{2 \alpha_{7, \infty 0} \Delta z}{2-\alpha_{7, \infty 0} \Delta z}\right.$ \\
\hline$\alpha_{4,00}=\left[\frac{\alpha_{1, C 0}}{\Delta z^{2}}-\frac{\alpha_{3, C 0}}{2 \Delta z}\right.$ & $\alpha_{10, \infty 0}=\left[\frac{2-\alpha_{3, \infty 0} \Delta z}{2+\alpha_{3, \infty 0} \Delta z}\right]$ \\
\hline$\alpha_{s, \infty 0}=\left[\frac{\alpha_{1, \infty 0}}{\Delta z^{2}}+\frac{\alpha_{3, \infty 0}}{2 \Delta z}\right.$ & $\alpha_{11, \infty 0}=\left[\frac{2 \alpha_{7, c_{0} \Delta z} \Delta z}{2+\alpha_{7, \infty 0} \Delta z}\right]$ \\
\hline$\alpha_{6, \infty}=\frac{2 \alpha_{1, \infty}}{\Delta z^{2}}$ & $\begin{array}{l}\alpha_{12, \mathrm{CO}}=\alpha_{4, \mathrm{CO}} \alpha_{8, \mathrm{CO}}+ \\
\alpha_{5, \mathrm{CO}} \alpha_{10, \mathrm{CO}}-\alpha_{6, \mathrm{CO}}\end{array}$ \\
\hline \multicolumn{2}{|c|}{$\alpha_{13, \infty 0}=\alpha_{5, C 0} \alpha_{11, \infty 0}+\alpha_{2, \infty 0}-\alpha_{4, \infty 0} \alpha_{9, \infty 0}$} \\
\hline
\end{tabular}

